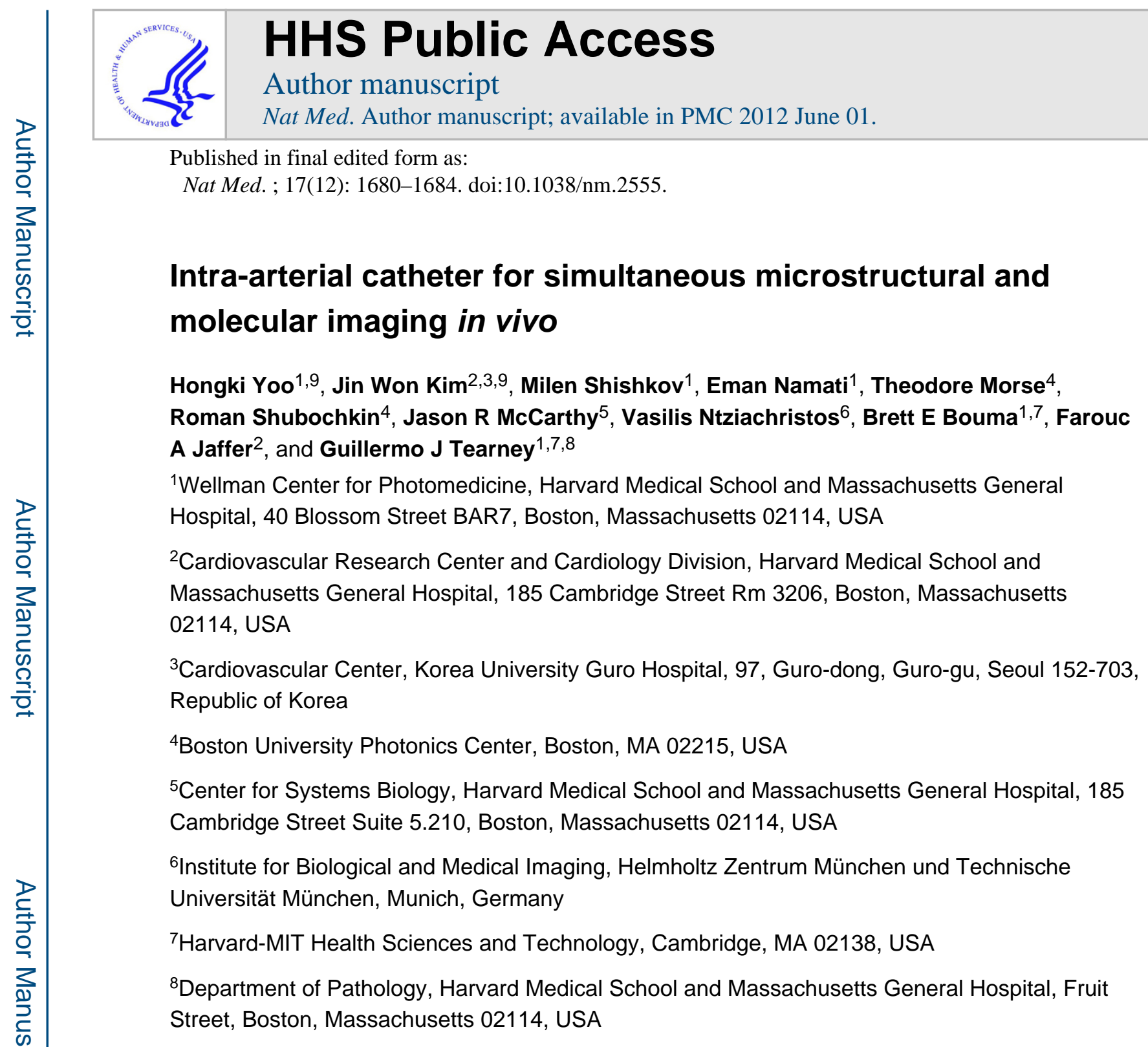

Abstract

Advancing our understanding of human coronary artery disease requires new methods that can be used in patients for studying atherosclerotic plaque microstructure in relation to the molecular mechanisms that underlie its initiation, progression, and clinical complications, including myocardial infarction and sudden cardiac death. Here we report a dual-modality intra-arterial catheter for simultaneous microstructural and molecular imaging in vivo using a combination of

\footnotetext{
Users may view, print, copy, download and text and data- mine the content in such documents, for the purposes of academic research, subject always to the full Conditions of use: http://www.naturecom/authors/editorial_policies/license.html\#terms

Correspondence : F.A.J. (fjaffer@mgh.harvard.edu) and G.J.T. (gtearney@partners.org).

9 These authors contributed equally to this work.

AUTHOR CONTRIBUTIONS

H.Y. developed dual-modality system and catheter and wrote the manuscript. H.Y. and J.W.K. designed and performed the experiments. H.Y., J.W.K., F.A.J. and G.J.T. analyzed and processed the data. M.S. contributed to catheter development. E.N. contributed to OFDI technology development. T.M. and R.S. designed and manufactured double-clad fiber. J.R.M. synthesized the fibrin-targeted nanoagents. V.N. contributed to the design of experiments and development of the animal model protocols. B.E.B. contributed to OFDI technology development. F.A.J. and G.J.T. contributed to the design of experiments, preparation of the manuscript and supervised the overall project. All authors read and edited the manuscript.
} 
optical frequency domain imaging (OFDI) and near-infrared fluorescence (NIRF) imaging. By providing simultaneous molecular information in the context of the surrounding tissue microstructure, this novel catheter could provide new opportunities for investigating coronary atherosclerosis and stent healing, and for identifying high-risk biological and structural coronary arterial plaques in vivo.

Atherosclerosis is an inflammatory, progressive disease of the arteries that accounts for the majority of deaths in the United States ${ }^{1}$. One of the most significant contributors to this high mortality rate is coronary artery disease (CAD), characterized by the buildup of atherosclerotic plaques within the coronary wall. These lesions can progress over time and may eventually precipitate clot formation (thrombosis), leading to heart attack (myocardial infarction) and sudden cardiac death. Due to the high prevalence of CAD, it is imperative that new in vivo tools are developed to investigate how coronary plaques evolve, disrupt blood flow, and cause heart attacks, and how they respond to different forms of treatment.

Since many of the underlying structural and molecular mechanisms involved in CAD occur on a microscopic scale, catheter-based high-resolution imaging techniques are ideal candidates for research and diagnosis in patients. Optical frequency domain imaging $(\mathrm{OFDI})^{2}$, also known as Frequency-domain optical coherence tomography (FD-OCT) ${ }^{3-6}$, has been shown to rapidly acquire three-dimensional (3D) images of the artery wall at a microscopic resolution within a few seconds ${ }^{2,7}$. Due to its superior resolution and high frame rates, intracoronary OFDI and FD-OCT has now emerged as one of the most promising new clinical methods for interrogating the microstructural detail of the coronary wall ${ }^{7,8}$. In addition, complementary cellular and molecular information in arterial disease, such as inflammatory protease activity, the macrophage composition of the inflammatory infiltrate, upregulated vascular cell adhesion molecules, and the presence or absence of fibrin, may be obtained by near-infrared fluorescence (NIRF) molecular imaging approaches ${ }^{9-13}$. Recently, an intravascular NIRF sensing approach has been successfully demonstrated for one-dimensional intravascular detection of atheroma inflammation in coronary-sized vessels in vivo using a protease-activatable NIRF agent ${ }^{9}$. While two techniques of OFDI and NIRF have been independently established, combination of both technologies in a single coronary catheter would greatly enhance their utility by visualizing molecular detail, precisely co-registered onto the microscopic architectural morphology of the artery wall, including following implantation of bare metal or drug-coated stents. Here, we present novel catheter-based, dual-modality, intra-arterial images simultaneously obtained with fully co-registered OFDI and NIRF in vivo.

\section{RESULTS}

\section{Dual-modality catheter imaging system}

Coincident microstructural and fluorescent molecular imaging was conducted with a dualmodality catheter system developed in our laboratory (Fig. 1). The catheter's outer transparent sheath $(800 \mu \mathrm{m}$ diameter; $2.4 \mathrm{~F})$ was the same as that used in ongoing singlemodality OFDI clinical studies ${ }^{7}$, thus facilitating the translation of this technology to patients in the future. A unique optical imaging probe, contained within the catheter's 
sheath, was constructed from a double-clad fiber that has a single-mode core that transmits and receives the OFDI light and a multi-mode light-guiding inner cladding that transmits the NIRF excitation and receives the emitted fluorescence light (Supplementary Fig. 1a) ${ }^{14}$. A side-viewing ball-lens, fabricated at the distal end of the fiber (Supplementary Fig. 1b,c), provided focused, co-registered OFDI and NIRF excitation spots within the artery wall. An OFDI depth profile (A-line) of the tissue's microstructure and a single fluorescence emission value was obtained at each imaging probe position. Three-dimensional microstructural OFDI and two-dimensional NIRF data was simultaneously obtained by helical pullback scanning of the imaging probe within the transparent catheter sheath (Supplementary Methods and Supplementary Video 1). The dual-modality system developed for the study provided microscopic cross-sectional OFDI images $(\sim 7 \mu \mathrm{m}$ axial resolution, $\sim 30 \mu \mathrm{m}$ transverse resolution) of tissue structure with a high frame rate (25.4 fps with 2048 radial scans per image), a long ranging depth (4.6 $\mathrm{mm}$ in saline), and high sensitivity $(110 \mathrm{~dB})$. As with other optical techniques, the penetration depth of both OFDI and NIRF are limited, but these techniques are still capable of obtaining detailed information on the first several millimeters of the artery wall.

The system simultaneously acquired molecular information from NIR fluorochromes with a lateral resolution of approximately $100 \mu \mathrm{m}$ at a sampling rate equal to the A-line acquisition rate of OFDI ( $52 \mathrm{kHz})$. The NIRF provided highly sensitive detection of less than $1 \mathrm{nM}$ of Cy7 NIR fluorochrome and showed a linear relationship to the concentration of the fluorochrome. Fluorochrome concentration on OFDI-NIRF images was quantified using both titration and distance calibration studies (Supplementary Methods and Supplementary Fig. 2). OFDI data was rendered for $3 \mathrm{D}$ visualization by segmenting the luminal wall ${ }^{15}$, stent struts ${ }^{16}$, and thrombus ${ }^{17}$ using previously established criteria for OCT image characterization ${ }^{7}$. Data from each modality was fused by mapping the NIRF signal onto the volume-rendered OFDI dataset (Supplementary Methods and Supplementary Fig. 3). The variation of the co-registration of OFDI and NIRF was measured to be less than $10 \mu \mathrm{m}$ in the rotational direction and $18 \mu \mathrm{m}$ in the longitudinal direction (Supplementary Methods and Supplementary Fig. 4), which is below the lateral resolution of OFDI.

\section{Intravascular imaging of stent-microthrombosis}

Stent-microthrombosis was one of the models chosen to validate this technique because identification of fibrin coverage on stents may provide information on the future risk of clinical stent thrombosis ${ }^{18,19}$. While OFDI can identify large protruding thrombi on a structural basis ${ }^{17}$, the greater biological specificity and sensitivity afforded by NIRF molecular imaging of fibrin may enable specific discrimination of key thrombus-associated molecules that overlie stent struts following implantation, a capability that is currently not possible with standalone OCT or OFDI ${ }^{20,21}$.

In our first experiment with the catheter, we performed dual-modality imaging of a cadaveric coronary artery with an implanted NIR fluorescent-fibrin labeled stent in vitro (Supplementary Methods and Fig. 2). Cross-sectional OFDI images of the stented cadaver coronary artery were comparable to standalone OFDI images ${ }^{7}$, clearly showing the microstructural detail of the arterial wall, metallic stent struts, and thrombus (Fig. 2a,b). The 
simultaneously acquired intravascular NIRF data was displayed as a cylinder, using maximum intensity projection volume rendering, (Fig. 2c) and displayed adjacent to fluorescence reflectance imaging (FRI) of the excised specimen (Fig. 2d). Side-by-side comparison of FRI and NIRF demonstrated a good visual correspondence between the intravascular NIRF representation and the FRI standard ${ }^{11}$.

To demonstrate the potential of dual-modality intra-arterial imaging of stent microthrombi in vivo, we acquired 3D datasets of a coronary stent that was covered by fluorescently labeled fibrin-rich thrombus, deployed in the right iliac artery of a New Zealand white (NZW) rabbit, and imaged in vivo. Comprehensive OFDI and NIRF data was successfully acquired from a $12.5 \mathrm{~mm}$-long segment of the iliac artery of a living NZW rabbit in $5 \mathrm{~s}$ with a frame interval of $100 \mu \mathrm{m}$. OFDI images demonstrated the microstructure of the rabbit artery, including metallic stent struts and thrombus (Fig. 3a). The simultaneously acquired NIRF signal (Fig. 3b) provided highly sensitive detection of the Cy7 NIR fluorochrome binding to fibrin. Comprehensive two-dimensional intra-arterial NIRF imaging provided sufficient spatial resolution to allow the visualization of stent struts (width $=81 \mu \mathrm{m}$ ) when surrounded by a strong fluorescent signal (Supplementary Fig. 5). When fused onto the luminal surfaces of the OFDI cross-sectional images, regions of the artery containing thrombi as identified by OFDI also displayed strong fluorescence from Cy7 labeled fibrin ${ }^{17}$ (red inset in Fig. 3c). NIRF signals were detected in other portions of the artery that did not contain clear OFDI evidence of thrombus (yellow inset in Fig. 3c), demonstrating an enhanced fibrin sensitivity for NIRF molecular imaging. Microscopic NIRF signal and fibrin-positive histology were highly correlated. The colocalization coefficients of OFDI-delineated thrombus and NIRF labeled fibrin were measured to be 0.98 and 0.67, respectively (Supplementary Methods and Supplementary Fig. 6). These coefficients, along with Figure 3, can be explained by the presence of fibrin in nearly all OFDI-delineated thrombi and a higher sensitivity of NIRF for detecting fibrin than structural characterization by OFDI alone. Color-coded volume rendering (Fig. 3d) and a flythrough movie (Supplementary Video 2) of the OFDI data with and without the NIRF overlay demonstrated that OFDI-delineated thrombus and Cy7 labeled fibrin were highly colocalized in three dimensions as well. Dual-modality intravascular imaging of the control, unstented aorta of the same rabbit demonstrated negligible NIR fluorescence, and the absence of OFDI-delineated thrombus (Supplementary Fig. 7).

\section{Intravascular imaging of inflammation in atherosclerosis}

To further demonstrate the clinical potential of dual-modality OFDI-NIRF imaging, simultaneous imaging of atherosclerotic plaque morphology and inflammation-associated enzymatic activity was performed in vivo. We generated atherosclerotic plaques in four NZW rabbits by the combination of balloon-denudation in the right iliac artery and the aorta, and a high cholesterol diet (7-9 weeks). The atheroma-bearing rabbits were intravenously injected with a cysteine protease-activatable NIRF agent 24 hours prior to imaging. The agent, validated in mouse and rabbit models of atherosclerosis, reports on the activity of cysteine proteases, including cathepsin B, in inflamed arteries ${ }^{9,22}$. Using the dual-modality intra-arterial catheter, simultaneous OFDI and NIRF images of normal and atherosclerotic arteries were acquired from both iliac arteries and the aorta in vivo. We obtained dualmodality images from a total of ten arteries: four in the aorta, three in the injured right iliac 
arteries and three in the non-injured left iliac arteries. A total of three pullbacks were performed in each artery. In two of the iliac arteries, we were unable to insert the catheter because of stenosis due to a high atherosclerotic burden or complex anatomical constraints.

Focal plaques were detected by OFDI-NIRF in the dataset that was acquired in $8 \mathrm{~s}$ from a 40 mm-long segment of the iliac artery (Fig. 4). Cross-sectional OFDI images showed the microstructure of the rabbit iliac artery with protruding focal plaque evident from approximately 3-6 o'clock (Fig. 4a). From the OFDI image, the focal plaque was characterized by morphological features, including a highly scattering, raised, thickening of the artery wall (Fig. 4a, arrowheads) overlying a signal poor region that is consistent with lipid-rich tissue (Fig. 4a, L) ${ }^{15}$. Co-localized NIRF obtained from the same region in vivo demonstrated a high NIR fluorescence signal, indicative of cysteine protease activity, whereas NIRF from other uninvolved portions of the artery wall was weak (Fig. 4b,c). RAM-11 stained sections (Fig. 4d) confirmed the OFDI findings of a plaque containing macrophages (Fig. 4d, arrowheads). Fluorescence microscopy of atheroma sections and NIR fluorescence (red channel) showed a high degree of spatial correspondence with the NIRF signals obtained in vivo (Fig. 4e). The cathepsin B signal was also high in the plaque as seen by immunohistochemistry (Fig. 4f), and the spatial pattern of cathepsin B staining corresponded to that of the NIRF signals obtained in vivo. Interestingly, the NIRF signal in the plaque was heterogeneous, with higher fluorescence around 4 o'clock (Fig. 4c, green asterisk) compared to that at 5 o'clock (Fig. 4c, blue asterisk). This finding, confirmed by NIR fluorescence microscopy and cathepsin B immunohistochemistry (Fig. 4h,i, green and blue asterisks), suggests that there may be differences in protease production and activity across varying populations of macrophages in this atherosclerotic plaque. The fact that this heterogeneity of inflammatory protease activity cannot be appreciated by OFDI alone demonstrates the power of this combined modality to provide complementary information on the presence of atherosclerosis and microstructural morphology of plaque by OFDI and inflammatory protease activity by NIRF imaging.

We further validated this technique's capability to measure plaque microstructure and protease content by comparing multiple $(n=13)$ cross-sectional OFDI-NIRF images from the four rabbits, obtained in vivo, with corresponding $\mathrm{H} \& \mathrm{E}$ and cathepsin $\mathrm{B}$ immunohistochemically-stained histologic sections (Supplementary Methods and Supplementary Fig. 8). Linear regression showed that OFDI and histologic measurements of percent plaque circumference were very highly correlated $(r=0.97, P<0.0001$;

Supplementary Fig. 8g). We also found a high correlation between the average NIRF signal intensity and cathepsin B immunostain-positive percent area $(r=0.82, P=0.0004$; Supplementary Fig. $8 \mathrm{~h}$ ). In order to assess the reproducibility of dual-modality imaging in vivo, we compared corresponding OFDI-NIRF cross-sections from paired test-retest pullback datasets. OFDI measurements of percent plaque circumferences for test and retest pullbacks were very highly correlated $(r=0.99, P<0.0001$; Supplementary Fig. 8i), showing an excellent OFDI measurement reproducibility, similar to that previously reported in clinical OCT studies ${ }^{23-25}$. Likewise, the values for the average NIRF signal intensity were very highly repeatable $(r=0.93, P<0.0001$; Supplementary Fig. $8 \mathrm{j})$, demonstrating reproducible NIRF measurement of molecular activity. 


\section{DISCUSSION}

The ability of this new technology to simultaneously provide colocalized microstructural and biological image information could prove useful for future understanding and management of both coronary artery disease and vessel wall healing following coronary stent implantation. Inflammatory cells, including macrophages, identified by $\mathrm{OFDI}^{7}$ can now be further characterized in vivo in terms of molecular expression and activity by use of cathepsin protease-activatable NIR fluorescent molecular beacons such as Prosense ${ }^{9,11}$. This new capability could allow researchers to gain knowledge on macrophage activity patterns for distinct plaque types and in response to different pharmacologic therapies. The ability of OFDI-NIRF to identify areas of active inflammation in the context of other microstructural features such as thin fibrous caps over lipid cores should increase the predictive ability of imaging for detecting vulnerable plaques in patients. Intraplaque angiogenesis, also implicated in vulnerable plaque ${ }^{26}$, may be imaged by use of near-infrared fluorescent molecular imaging agents targeted to integrins, such as $\alpha_{\mathrm{v}} \beta_{3}{ }^{13}$. Drug eluting stents, commonly used to treat ischemic coronary disease, prevent restenosis by attenuating smooth muscle proliferation. These devices unintentionally may also prevent endothelial regrowth, leading to fibrin deposition ${ }^{20}$ and uncommon, but devastating or fatal occurrences of stent thrombosis ${ }^{18}$. While standalone intracoronary OCT and OFDI are currently being investigated for the evaluation of stent tissue coverage in patients ${ }^{16,21}$, these imaging technologies are limited by their inability to determine whether or not the tissue overlying stent struts are fibrin, platelets, neointima, endothelial or inflammatory cells. Simultaneous intra-arterial molecular-structural imaging in a rapid, single pullback could provide this critical information that is required to determine whether or not patients who receive drug coated stents need to remain on protracted anti-clotting medications ${ }^{18,20,21}$ to prevent these potential complications. It is likely that many of these research and clinical applications of this multimodality technology can be realized soon; the dual-modality catheter presented here is mechanically and structurally identical to standalone OFDI catheters currently used in the cardiac catheterization laboratory ${ }^{7}$ and FDA-approved NIR fluorescence imaging agents, such as Indocyanine Green, and molecular-activity agents in the regulatory approval pipeline like Prosense, are anticipated to be available for human coronary imaging in the near term.

\section{METHODS}

\section{System development and signal processing}

OFDI provides high-resolution images of tissue by processing interference signals of light reflected from the sample and a reference. The NIRF imaging system was developed for detecting NIRF molecular imaging agents such as Prosense. We created a dual-modality catheter and rotary junction based on a double-clad fiber for simultaneous OFDI and NIRF imaging. The design and construction of the OFDI-NIRF system, including the dualmodality rotary junction and catheter, and the methods for image processing and visualization are described in the Supplementary Methods. 


\section{Imaging of fibrin-rich thrombi on a coronary stent in vivo}

We conducted arterial imaging of a thrombus-covered stent in one NZW rabbit. Rabbit arteries were chosen in this study, since rabbit aorta and iliac arteries have average diameters of 3-3.5 and 2-2.5 mm respectively, which are comparable to diameters of human coronary arteries. Before the in vivo procedure, we prepared the NIRF fibrin-coated stent as described in the Supplementary Methods. Subsequently, we deployed the stent into an iliac artery of an anaesthetized rabbit weighing approximately $3 \mathrm{~kg}$. First, we inserted a long sheath into the left carotid artery. A guide wire (0.014") was inserted and advanced down to the right iliac artery. The coronary stent was inserted at the right iliac artery and deployed at $10 \mathrm{~atm}$. The procedure was guided by $\mathrm{x}$-ray angiography. After stent deployment, we inserted a $6 \mathrm{~F}$ Proxis catheter (St. Jude medical) for saline flushing and guiding of the imaging catheter. The OFDI-NIRF imaging catheter was inserted and placed near the distal end of the stent using angiographic visualization of radiopaque markers present on the dual-modality catheter ${ }^{7,9}$. Real-time cross-sectional OFDI images and NIRF signals were monitored, while the imaging probe was rotating at a speed of $25.4 \mathrm{rps}$. Blood was removed by manual flush of a $20 \mathrm{ml}$ bolus of saline through the Proxis catheter at a rate of approximately $1.0 \mathrm{ml} \mathrm{s}^{-1}$. As soon as the field of view was cleared, pullback was initiated and both the OFDI and NIRF signals were recorded to hard drives. The pullback and recordings were terminated after imaging over a pre-defined length. A total of nine pullbacks were performed on the right iliac artery with different pullback speeds $\left(2.5-10.0 \mathrm{~mm} \mathrm{~s}^{-1}\right)$ and different flushing methods (saline flushing vs. no flushing). In three pullbacks performed without flushing, the OFDI view was totally obstructed by the blood while NIRF signal was still acquired with a decreased intensity and degraded spatial resolution. In one pullback with flushing, flushing did not provide clear viewing of the artery wall. We successfully acquired the volumetric dual-modality information of $12.5 \mathrm{~mm}$-long-segment from the remaining five pullbacks with flushing. Various pullback speeds did not affect OFDI or NIRF image quality. After imaging of the stented right iliac artery, we performed 2 pullbacks on the native, unstented aorta of the same rabbit as a control with different pullback speeds $\left(2.5\right.$ and $\left.5.0 \mathrm{~mm} \mathrm{~s}^{-1}\right)$ with saline flushing. Volumetric data sets of $30 \mathrm{~mm}$-long-segments were successfully acquired. The Hospital's subcommittee on research animal care (SRAC) approved all animal protocols.

\section{Atherosclerotic plaque model and imaging in vivo}

To image atheroma structure and inflammation in vivo, four NZW rabbits (weight, 3-3.5 kg) were placed on a high-cholesterol diet (1\% cholesterol and 5\% peanut oil, C-30293, Research Diets, Inc) for 1 week prior to balloon injury. Anesthesia was induced and continued during the procedure. Then, we inserted a 3 F Fogarty arterial embolectomy catheter (Edwards Lifesciences) into the left carotid artery and advanced it down to the right iliac artery. Next, the balloon was inflated to tension (2-6 atm) and three pullbacks were performed in the right iliac artery and the proximal infra-renal aorta. Following injury, the rabbits were continued on the high-cholesterol diet. After 6-8 weeks, the rabbits were injected with a NIRF protease-activatable agent (Prosense750 VM110, VisEn Medical, 600 $\left.\mathrm{nmol} \mathrm{kg}{ }^{-1}\right)^{9,22}$. At 24 hours post-injection, anesthesia was induced and a guiding sheath was inserted into the right carotid artery to the abdominal aorta under x-ray angiographic guidance. A Proxis sheath was then inserted through the guiding sheath down to the imaging 
position following the insertion of the guide wire (0.014"). The imaging catheter was inserted through the Proxis sheath and placed at the imaging position. We performed imaging pullbacks with a balloon-occlusive flushing $\left(1 \mathrm{ml} \mathrm{s}^{-1}\right)$ and pullback speeds of 5 or $10 \mathrm{~mm} \mathrm{~s}^{-1}$. A total of three pullbacks were conducted for each artery. We successfully acquired volumetric datasets in $4-15 \mathrm{~s}$ from $40-75 \mathrm{~mm}$-long segments of the arteries. The frame rate was $25.4 \mathrm{fps}$ and the frame interval was 200 or $400 \mu \mathrm{m}$.

Histology

Detailed information on the histopathologic methods can be found in the Supplementary Methods section.

\title{
Quantitative Analysis
}

The quantitative analysis methods are described in detail in the Supplementary Methods section.

\section{Supplementary Material}

Refer to Web version on PubMed Central for supplementary material.

\section{ACKNOWLEDGEMENTS}

\begin{abstract}
We thank J. Gardecki for preparation of the cadaver coronary artery and CVPath for pathology of the stented artery. We also thank A. Rosenthal and G. Mallas for their technical support, A. Mauskapf for preparing and assisting animal procedures. We thank Y. Iwamoto, Y. Yagi and E. Salomatina for assistance in histopathology.

This research was supported in part by US National Institutes of Health (contracts R01HL076398 and R01HL093717, G.J.T., R01HL108229-01A1, F.A.J.), by Center for Integration of Medicine and Innovative Technology (contract DAMD17-02-2-0006, G.J.T., F.A.J.), an American Heart Association Scientist Development Grant (\#0830352N, F.A.J.), Howard Hughes Medical Institute Early Career Award (F.A.J.), and Cardio Vascular Research Foundation (CVRF, J.W.K.).
\end{abstract}

\section{REFERENCES}

1. Lloyd-Jones D, et al. Heart disease and stroke statistics--2009 update: a report from the American Heart Association Statistics Committee and Stroke Statistics Subcommittee. Circulation. 2009; 119:480-486. [PubMed: 19171871]

2. Yun SH, et al. Comprehensive volumetric optical microscopy in vivo. Nat Med. 2006; 12:1429_ 1433. [PubMed: 17115049]

3. Huang D, et al. Optical coherence tomography. Science. 1991; 254:1178-1181. [PubMed: 1957169]

4. Choma M, Sarunic M, Yang C, Izatt J. Sensitivity advantage of swept source and Fourier domain optical coherence tomography. Opt Express. 2003; 11:2183-2189. [PubMed: 19466106]

5. Hausler G, Lindner MW. "Coherence Radar" and "Spectral Radar" - New tools for dermatological diagnosis. Journal of Biomedical Optics. 1998; 3:21-31. [PubMed: 23015002]

6. Fujimoto JG. Optical coherence tomography for ultrahigh resolution in vivo imaging. Nat Biotechnol. 2003; 21:1361-1367. [PubMed: 14595364]

7. Tearney GJ, et al. Three-dimensional coronary artery microscopy by intracoronary optical frequency domain imaging. JACC Cardiovasc Imaging. 2008; 1:752-761. [PubMed: 19356512]

8. Takarada $\mathrm{S}$, et al. Advantage of next-generation frequency-domain optical coherence tomography compared with conventional time-domain system in the assessment of coronary lesion. Catheter Cardiovasc Interv. 75:202-206. [PubMed: 19937788]

9. Jaffer FA, et al. Real-time catheter molecular sensing of inflammation in proteolytically active atherosclerosis. Circulation. 2008; 118:1802-1809. [PubMed: 18852366] 
10. McCarthy JR, et al. Multimodal nanoagents for the detection of intravascular thrombi. Bioconjug Chem. 2009; 20:1251-1255. [PubMed: 19456115]

11. Ntziachristos V, Bremer C, Weissleder R. Fluorescence imaging with near-infrared light: new technological advances that enable in vivo molecular imaging. Eur Radiol. 2003; 13:195-208. [PubMed: 12541130]

12. Sanz J, Fayad ZA. Imaging of atherosclerotic cardiovascular disease. Nature. 2008; 451:953-957. [PubMed: 18288186]

13. Jaffer FA, Libby P, Weissleder R. Optical and multimodality molecular imaging: insights into atherosclerosis. Arterioscler Thromb Vasc Biol. 2009; 29:1017-1024. [PubMed: 19359659]

14. Ryu SY, Choi HY, Na J, Choi ES, Lee BH. Combined system of optical coherence tomography and fluorescence spectroscopy based on double-cladding fiber. Opt Lett. 2008; 33:2347-2349. [PubMed: 18923618]

15. Yabushita $\mathrm{H}$, et al. Characterization of human atherosclerosis by optical coherence tomography. Circulation. 2002; 106:1640-1645. [PubMed: 12270856]

16. Takano M, et al. Evaluation by optical coherence tomography of neointimal coverage of sirolimuseluting stent three months after implantation. Am J Cardiol. 2007; 99:1033-1038. [PubMed: 17437723]

17. Kume T, et al. Assessment of coronary arterial thrombus by optical coherence tomography. Am J Cardiol. 2006; 97:1713-1717. [PubMed: 16765119]

18. Camenzind E, Steg PG, Wijns W. Stent thrombosis late after implantation of first-generation drugeluting stents: a cause for concern. Circulation. 2007; 115:1440-1455. discussion 1455. [PubMed: 17344324]

19. Holmes DR Jr, et al. Stent thrombosis. J Am Coll Cardiol. 2010; 56:1357-1365. [PubMed: 20946992]

20. Finn AV, et al. Vascular responses to drug eluting stents: importance of delayed healing. Arterioscler Thromb Vasc Biol. 2007; 27:1500-1510. [PubMed: 17510464]

21. Guagliumi G, Sirbu V. Optical coherence tomography: high resolution intravascular imaging to evaluate vascular healing after coronary stenting. Catheter Cardiovasc Interv. 2008; 72:237-247. [PubMed: 18655155]

22. Chen J, et al. In vivo imaging of proteolytic activity in atherosclerosis. Circulation. 2002; 105:2766-2771. [PubMed: 12057992]

23. Prati F, et al. Expert review document on methodology, terminology, and clinical applications of optical coherence tomography: physical principles, methodology of image acquisition, and clinical application for assessment of coronary arteries and atherosclerosis. Eur Heart J. 2010; 31:401-415. [PubMed: 19892716]

24. Terashima M, et al. Accuracy and reproducibility of stent-strut thickness determined by optical coherence tomography. J Invasive Cardiol. 2009; 21:602-605. [PubMed: 19901417]

25. Gonzalo N, et al. Reproducibility of quantitative optical coherence tomography for stent analysis. EuroIntervention. 2009; 5:224-232. [PubMed: 19527980]

26. de Boer OJ, van der Wal AC, Teeling P, Becker AE. Leucocyte recruitment in rupture prone regions of lipid-rich plaques: a prominent role for neovascularization? Cardiovasc Res. 1999; 41:443-449. [PubMed: 10341843]

27. Otsu N. A threshold selection method from gray-level histograms. IEEE Transactions on Systems, Man and Cybernetics. 1979; 9:62-66. 


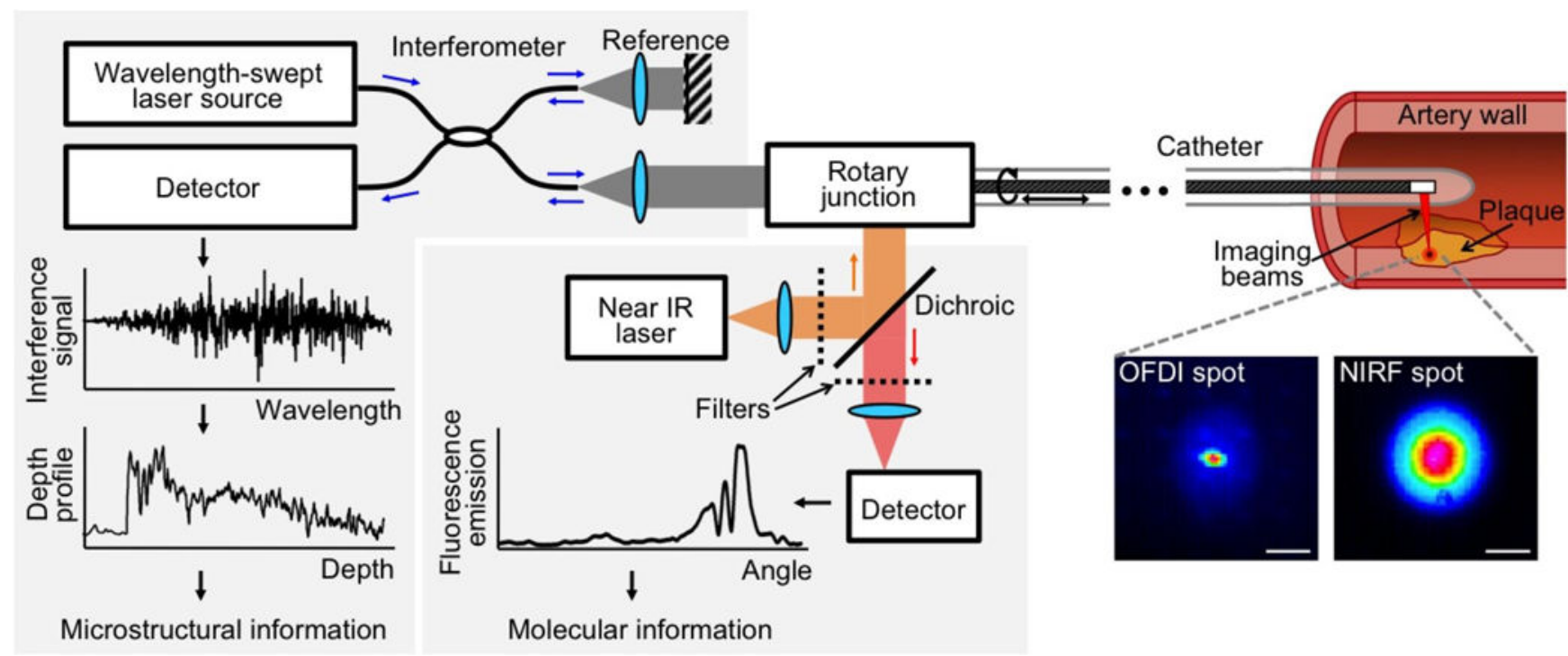

Figure 1.

Schematic of the dual-modality intra-arterial catheter for simultaneous microstructural and molecular imaging using optical frequency-domain imaging (OFDI) and near-IR fluorescence (NIRF). OFDI and NIRF systems are combined in one system by a dualmodality rotary junction that rotates and pulls back the imaging probe contained within the transparent catheter sheath, in a manner analogous to that used in standalone OCT, OFDI and intravascular ultrasound (IVUS). The imaging probe consists of a double-clad fiber that transmits OFDI and NIRF light through separate concentric light-guiding channels and focuses the beams into the sample. In the OFDI system, the light source changes its wavelength rapidly as a function of time. The depth profile (A-line) is reconstructed by taking the Fourier transform of the spectral interference signal that is generated by combining the reference and back-reflected signals from tissue microstructures. Depth profiles are continuously acquired to form cross-sectional images while the probe is rotating. NIR fluorescence emission, which reports molecular information, is also simultaneously acquired at every A-line acquisition. Scale bars, $100 \mu \mathrm{m}$. 

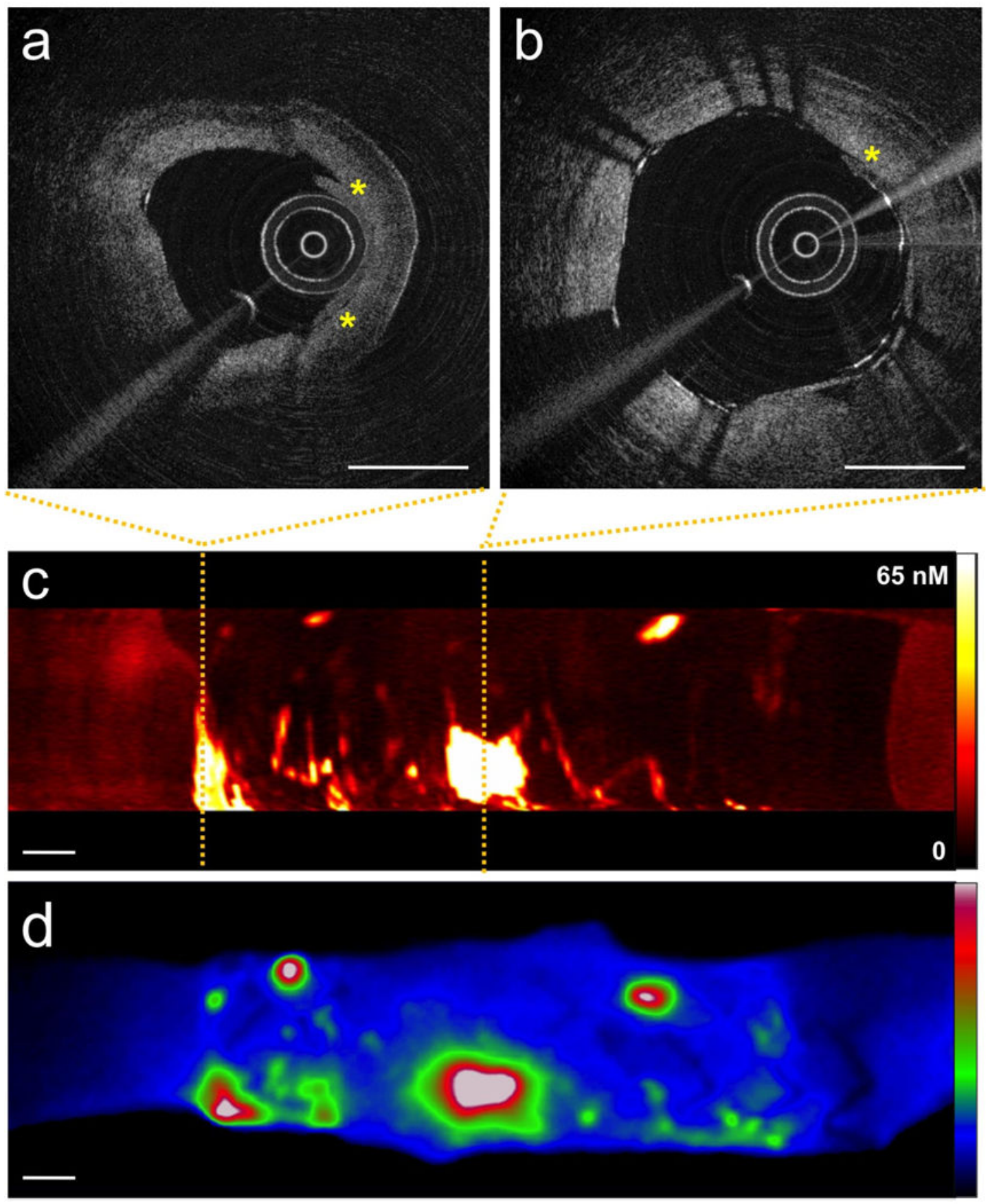

Figure 2.

Images of a cadaveric human coronary artery with a stent containing Cy7-labeled fibrin in vitro. (a) OFDI cross-sectional image with thrombus (yellow asterisks). (b) OFDI crosssectional image with stent struts and micro-thrombi (yellow asterisk). (c) NIRF cylindrical rendering of fibrin signal acquired by the dual-modality imaging catheter. (d) Fluorescence reflectance imaging (FRI) with Cy7 filter set. The FRI results strongly correspond to the dual modality OFDI-NIRF image. Scale bars, $1 \mathrm{~mm}$. 
a

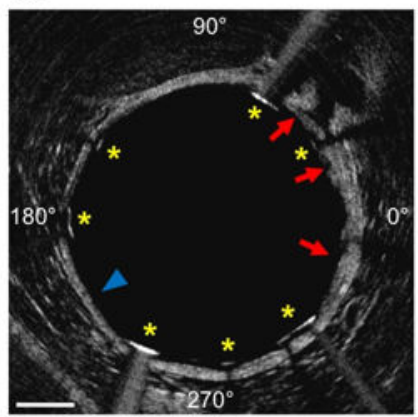

b

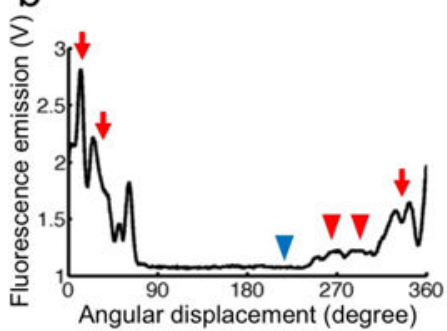

d

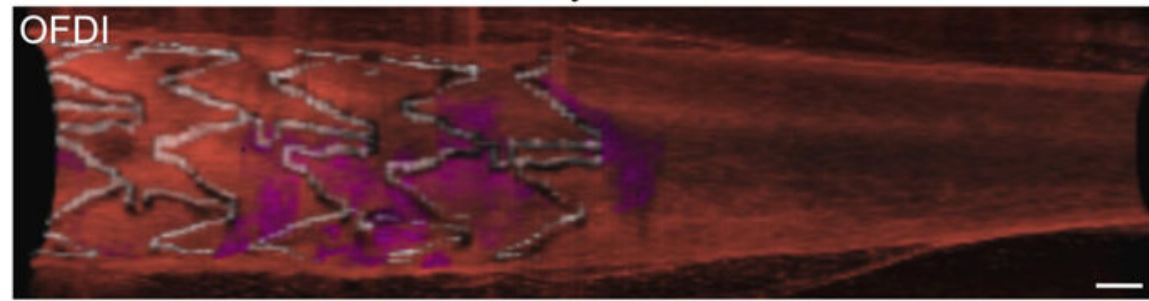

OFDI-NIRF
OFDI

C
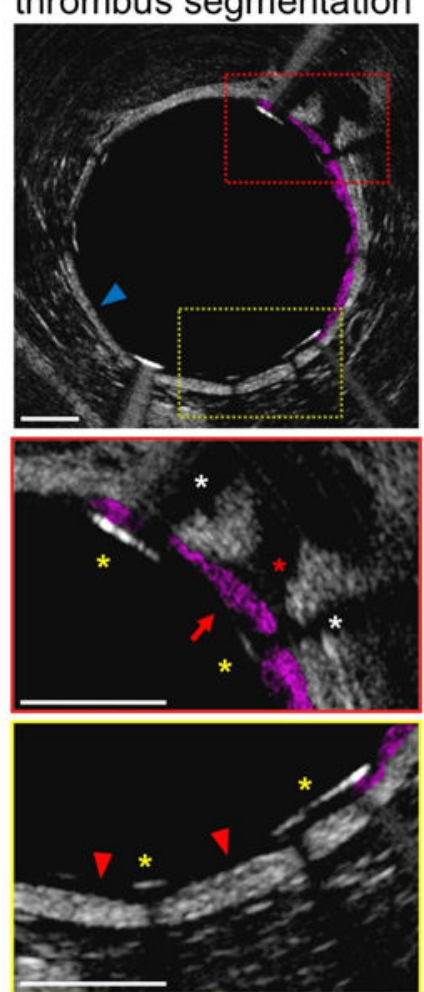

Cut-away view
OFDI-NIRF

\section{overlay}
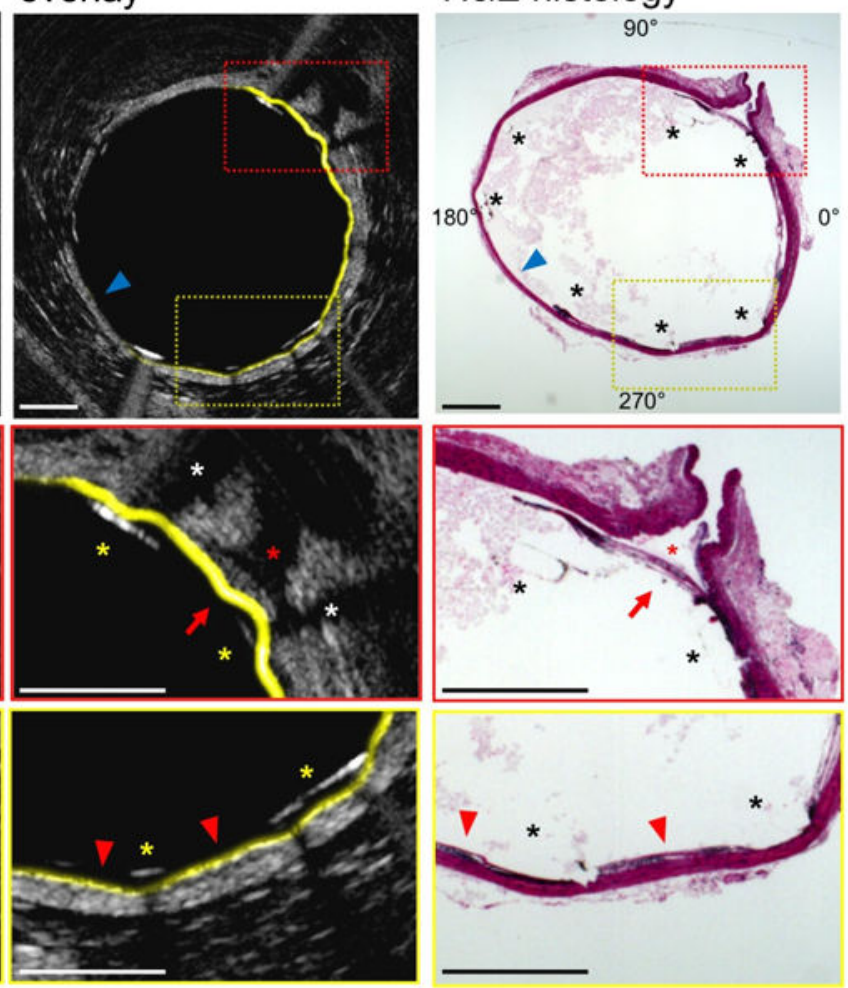

Flythrough view

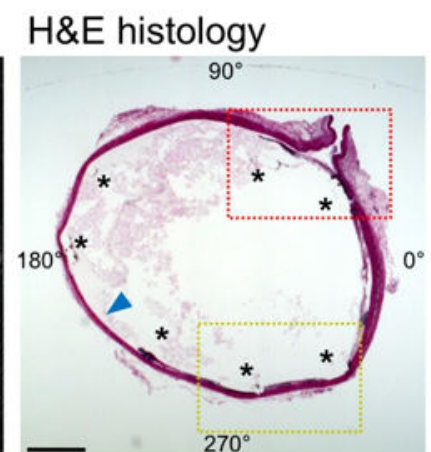

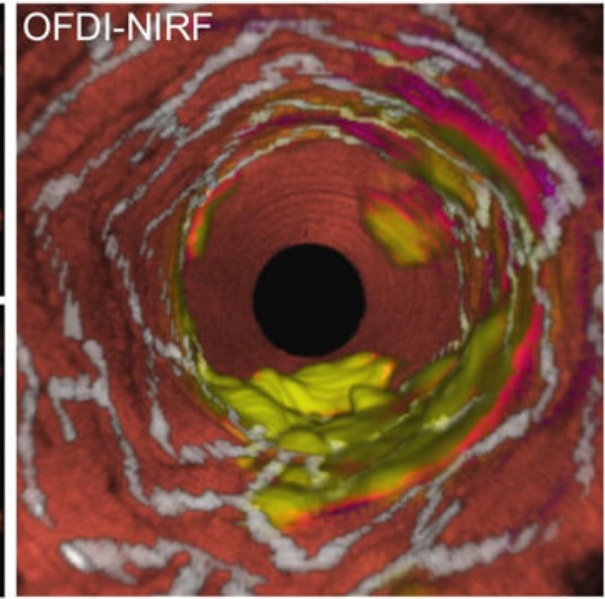

Figure 3.

Dual-modality OFDI-NIRF images of an iliac artery of a rabbit with an implanted NIR fluorescent fibrin-coated stent, in vivo. (a) A representative cross-sectional image of OFDI data. OFDI shows the cross-sectional arterial microscopic architectural morphology, including thrombus (red arrow) and stent struts (yellow asterisks). (b) A corresponding onedimensional signal of NIRF data simultaneously acquired with OFDI. The NIRF signal shows strong peaks where the thrombi were detected in OFDI (red arrows in a,b). The NIRF signal was negligible in areas that did not show OFDI evidence of thrombus or fibrin by histology (blue arrowhead in a,b) (c) OFDI (gray scale) with thrombus segmentation 
(purple) (left column), overlaid OFDI (gray scale) and NIRF (yellow scale) images (middle column), and corresponding H\&E histology (Original magnification $\times 25$ ) (right column). The top row and the insets demonstrate OFDI, NIRF, and histology data showing corresponding microstructural features, such as a side branch (red asterisk), stent struts (yellow asterisks in OFDI, black asterisks in H\&E), and thrombi (red arrow). Areas (blue arrowhead) without NIRF signal were devoid of fibrin by histology. Strong NIRF was observed where thrombus was detected by OFDI and histology (middle row, red arrow). In another area (lower row), a weaker fluorescence signal was detected corresponding to fibrin as confirmed by histology, but without evidence of protruding thrombus by OFDI (red arrowheads). White asterisks-stent strut shadows. (d) Three-dimensional rendering of the stented right iliac artery of a living rabbit. Structural components were segmented and colorcoded in OFDI images for clear visualization. Pink-artery wall; white-stent; purplethrombus; yellow-NIR fluorescent fibrin. Scale bars, $500 \mu \mathrm{m}$. 

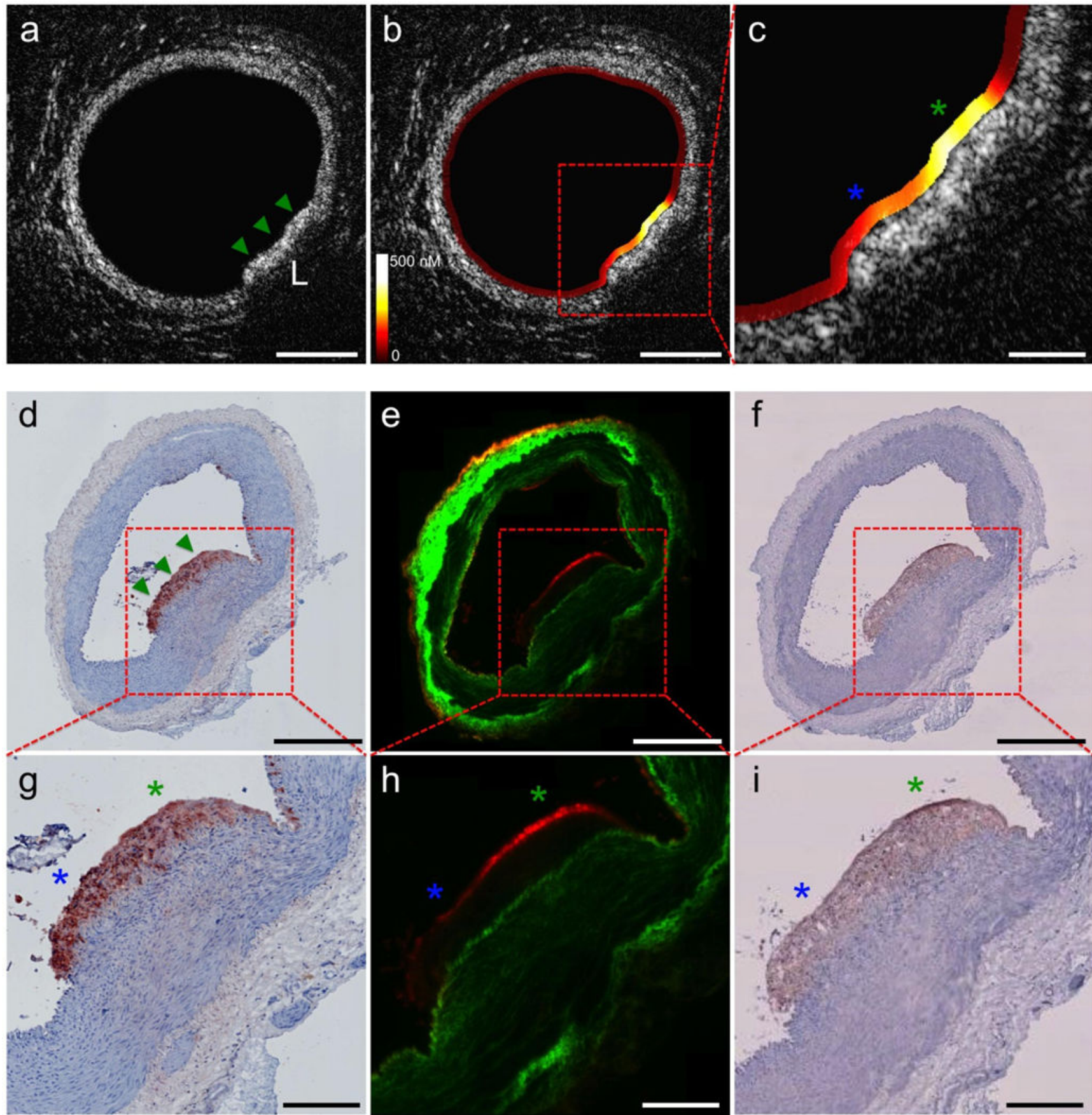

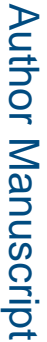

Figure 4.

Dual-modality OFDI-NIRF images of atherosclerosis microstructure and inflammatory enzyme activity in a living rabbit. (a) A representative OFDI cross-sectional image. OFDI shows normal artery wall as well as a focal, raised, lipid-containing (L) atherosclerotic lesion at 3-6 o'clock (arrowheads). (b) The OFDI-NIRF fusion image shows a strong NIRF signal in the OFDI-delineated plaque. The NIRF signal reflecting inflammatory protease activity was minimal in the area that appeared to be normal by OFDI. (c) Expanded view of b. 4 o'clock portion (green asterisk) of the plaque demonstrated a stronger NIRF signal than 
the adjacent regions (blue asterisk) (d) RAM-11 stained section demonstrates a plaque with a dense accumulation of macrophages. (e) Fluorescence microscopy image (20x, stitched) of autofluorescence (green) and protease activity-induced NIR fluorescence (red). Strong NIR fluorescence signal was detected at the luminal surface of the plaque, subtending a similar arc as that of the elevated NIRF signal obtained in vivo. (f) Immunoreactive cathepsin B was detected in the macrophage-rich plaque region. (g-i) Expanded views of $\mathbf{d}-\mathbf{f}$, respectively. Macrophage staining by RAM-11 did not appear to vary significantly across the plaque. However, as with NIRF signals in vivo, fluorescence microscopy and IHC for cathepsin B demonstrated heterogeneous protease activity in the plaque with a higher signal at 4 o'clock (green asterisk) compared with that at 5 o'clock (blue asterisk), similar to the pattern observed in the NIR fluorescence microscopic images in $\mathbf{h}$. Scale bars, $500 \mu \mathrm{m}(\mathbf{a}, \mathbf{b}, \mathbf{d}-\mathbf{f})$ and $200 \mu \mathrm{m}(\mathbf{c}, \mathbf{g}-\mathbf{i})$. 